EPOS, XVII (2001), págs. 13-27

\title{
IDENTIFICACIÓN DE DOS ESTADOS EN LA EDICIÓN DEL BELLUM GALLICUM DE JULIO CÉSAR (Burgos 1491) ${ }^{1}$
}

ANTONIO MoREno HeRnÁNDEZ Universidad Nacional de Educación a Distancia

\section{RESUMEN}

Entre las primeras ediciones incunables del Bellum Gallicum de Julio César se encuentra la que se imprimió en Burgos en 1491, en el taller de Juan de Burgos. De esta edición se conservan dos ejemplares cuyo estudio permite comprobar que no son idénticos, sino que presentan una significativa gama de variantes cuyo consideración constituye el principal objetivo de este artículo. De la colación y análisis de estas lecturas discrepantes se desprende que se trata de dos estados de la misma edición, cuya naturaleza se explica a partir de las técnicas de impresión y edición de los primeros incunables y cuya intención responde al deseo de ajustar el texto latino a la edición que sirve de base a la burgalesa: la edición veneciana de 1482 .

' Este artículo se ha realizado dentro del proyecto de Investigación «La recepción de César en España: bases filológicas para su estudio», que se viene desarrollando en el Departamento de Filología Clásica de la Universidad Nacional de Educación a Distancia. 
La técnica de edición de los textos clásicos latinos en España durante el período de los incunables es una parcela de la historia de la trasmisión que presenta todavía algunas vertientes escasamente conocidas, a falta de estudios específicos que suministren el análisis filológico del texto que portan muchas de estas primeras obras impresas ${ }^{2}$.

De la edición del Bellum Gallicum de Julio César realizada en 1491 en el taller de Juan de Burgos, cuyo estudio y filiación se ha llevado a cabo en otro lugar ${ }^{3}$, se conservan tan sólo dos ejemplares ${ }^{4}$ :

${ }^{2}$ Entre los estudios sobre la edición de incunables en España, resultan instrumentos imprescindibles los repertorios y trabajos de C. HAEBLER, The Early Printers of Spain and Portugal, Londres 1897; Typographie ibérique du quinzième siècle: reproductions en facsimile de tous les caractères typographiques employés en Espagne et en Portugal jusqu' à l'année 1500, La Haya-Leipzig 1901; Bibliografía ibérica del siglo XV: enumeración de todos los libros impresos en España y Portugal hasta el año de 1500, 2 vols., La Haya-Leipzig 1904-1917 reimp. Madrid 1992: FR. Vindel, El arte tipográfico en España durante el s. XV, 9 vols., Madrid 1945-1954; M. Chambers, «Early Printing and Book Illustration in Spain». The Library Chronicle 39, 1973, pp. 3-17; L. WITTEN, "The Earliest Books Printed in Spain», Papers of the Bibliographical Society of America 53, 1959, pp. 91-13: G. PAINTER, "General Introduction», Catalogue of Books Printed in the XVth century now in the British Museum, vol. X, Londres 1908-1971, pp. IX-XXXV: S. H. STEINBERG. Five Hundred Years of Printing, 3. ${ }^{a}$ ed., Harmondsworth 1974; Fr. García CRaviotto (coord.), Catâlogo General de Incunables en Bibliotecas Españolas, Madrid 1989, p. 245, n. ${ }^{\circ}$ 1585; El Catálogo Colectivo del Patrimonio Bibliográfico Español, del Ministerio de Educación. Cultura y Deporte; Gesamtkatalog der Wiegendrucke VI, Stuttgart-Nueva York, 1934 (1968) p. 31, n. ${ }^{\circ} 5875$; para los comienzos del XVI, cf. F. J. NorTon, La imprenta en España, 1501-1520. Madrid 1996; A descriptive Catalogue of Printing in Spain and Portugal 1501-1520, Cambridge 1978; sobre la impresión de traducciones de autores clásicos en España, cf. TH. S. BEARDSLEY, «Spanish Printers and the Classics: 1482-1599», Hispanic Review 47, 1979, pp. 25-35, así como su libro Hispano-Classical Translations between 1482 and 1699, Pittsburgh-Lovain 1970. Un panorama general sobre la transición de los manuscritos a las primeras ediciones incunables puede verse en M. DAviES, «El libro humanístico en el Cuatrocientos», en J. KRAYE, Introducción al humanismo renacentista. (ed. española a cargo de C. Clavería), Cambridge 1998, pp. 73-92.

3 A. Moreno Hernández, «La edición incunable del Bellum Gallicum de Julio César de Burgos 1491 », Cuadernos de Filología Clásica, (Estudios Latinos), 22, 2002, en prensa.

4 Fr. Méndez y C. HAEbler afirman haber conocido un único ejemplar de esta edición que estaría encuademado junto con la obra de Lucio MARINEO Sículo De Hispaniae Laudibus (cf. Fr. MÉndez, Tipografía española, Madrid 1866 (2. ${ }^{\circ}$ ed.), p. 135 (9); C. HAEBLER, Bibliografía ibérica del siglo XV.op. cit., n. ${ }^{\circ} 112$ [p. 50] afirma:»Este libro raro existe en la BNM; el ejemplar que allí manejé es el mismo que vio MÉndez encuadernado con el de Marineo, De Hispaniae laudibus. No se conoce otro ejemplar»). MENÉndez PELAYo se refiere también al ejemplar de la Biblioteca de la Universidad Complutense, y se refiere también al de la Nacional, añadiendo el dato de que el texto de Cesar estaba "encuadernado al fin de una obra de MARINeo SiCulo, De Hispaniae laudibus» (Bibliografia Hispano Latina Clásica, Santander-Madrid 1902 [1950].vol. II, p. 115). FR. VINDEL. en cambio, se refiere al libro como un volumen exento (El arte tipográfico en España durante el s. $X V$, Burgos y Guadalajara, vol VII), Madrid 1951, pp. 60-64 (n. 20). En los fondos registrados actualmente en los catálogos de la Biblioteca Nacional no se registra ningún otro ejemplar de la 
A) Biblioteca Nacional de Madrid, I/2067 (olim I/1879), ejemplar ${ }^{5}$ que identificaremos con la sigla $\mathbf{b}^{\mathbf{m}}$.

$50 \mathrm{ff}$. sin numerar, más dos de guarda iniciales y otra final. Contiene los libros I-VII del B.G. Encuadernación moderna en pergamino, en el lomo $C$. Julii Caesaris commentaria. Sin ninguna indicación sobre su procedencia.

B) Biblioteca Histórica «Marqués de Valdecilla» (Universidad Complutense de Madrid), sign. I-275, identificado ${ }^{6}$ como $\mathbf{b}^{\mathrm{c}}$.

Volumen facticio que contiene: Marco Antonio Sabellico, Opera (Ep. Fam., Orat., Poem.) (Venecia 1502), seguido de los libros I-VII del B.G. (Burgos 1491). Encuadernado en pergamino. Guardas con fragmentos de un impreso de derecho civil, al parecer incunable e impreso por Baptista de Tortis ${ }^{7}$ Este volumen formó parte, antes de su emplazamiento actual, del fondo antiguo de la Facultad de Derecho de la Universidad Complutense, y presenta en margen inferior del f. $2^{r}$ la

edición de Juan de Burgos, además del que nos va a ocupar aquí (I/206), ni se consigna en ellos la existencia de algún otro volumen que contenga ambas obras. De la edición incunable de De Hispaniae laudibus impresa por Fadrique de Basilea c. 1497 (cf. HAEBLER 399; Vindel VII,53: CGIBE 3831 ) se conservan en la Biblioteca Nacional de Madrid seis ejemplares (sign. I/2429; I/922; $\mathrm{I} / 1242 ; \mathrm{V} / 1922 ; \mathrm{V} / 1967 ; \mathrm{I} / 2268$ ), pero actualmente se trata de códices que contienen exenta esta obra de MARineo Sículo.

Sobre la obra del impresor Juan de Burgos y el entorno de su producción, cf. J. A. SAGREDo FERnÁNDEZ, Fuentes para el estudio de la imprenta en Burgos, Madrid 1997; L. CUESTA GUTIERREZ, «La imprenta en Burgos a través de su historia», Gutenberg Jahrbuch 1943, pp. 83-99; D. Hergueta, «Los incunables burgaleses», Boletín de la Comisión Provincial de Monumentos Históricos y Artísticos de Burgos, IX, 30, 1930, pp. 105-113; «Nuevos datos sobre los incunables burgaleses», op. cit., XI. 42, 1933, 406-409; 437-441; F. J. NORTON, A Descriptive Catalogue of Printing in Spain and Portugal 1501-1520, Cambridge 1978, p. 111." y ss.; A. ODRIOZOLA, "La imprenta en Castilla en el s. XV», en Historia de la imprenta en España, Madrid 1982, pp. 91-220; J. Delgado Casado, Diccionario de impresores españoles (siglos XV-XVII), vol. I, Madrid 1996, pp. 101102.

5 Descrito en D. Garcia Rojo y G. Ortiz de Montalván, Catálogo de incunables de la Biblioteca Nacional de Madrid, Madrid 1945, n. ${ }^{\circ} 470$; Catálogo Colectivo del Patrimonio Bibliográfico Español (http://www.mcu.es/ccpb/index.html) código de control: CCPB000108820. L. Cuesta Rodriguez se refiere a este ejemplar de la Biblioteca Nacional como de edición «muy rara, acaso única», cf. «Incunables con grabados de la Biblioteca Nacional de Madrid», GutenbergJahrbuch 1935, 74-92 (p. 84).

- Descrito en J. CANTó y A. Huarte, Catálogo de incunables de la Biblioteca de la Universidad Complutense, Madrid 1998, p. 49, n. ${ }^{\circ} 169$.

7 Otros fragmentos en 1-3; 4, 19, 28, 35, 36, 138, 169, 190, 192, 203, 210, 213, 214, 230, 268, 269, 272, 275, 277, 293, 315, 324, 328. Cf. J. Cantó y A. Huarte, op. cit, p. 49. 
anotación manuscrita "Visto en 1614», así como un ex libris del Colegio Mayor de San Ildefonso de Alcalá de Henares, cuyos fondos se trasladaron a Madrid en 1841. La mayoría de los mismos fueron adquiridos en época de Cisneros y debían estar en el Colegio a principios del s. XVII ${ }^{8}$.

Pues bien, los estudios existentes ${ }^{9}$ hasta el momento identifican ambos ejemplares como fruto de la misma edición, dado que todos su datos externos editoriales, la tipografía gótica, la composición y colofón son iguales ${ }^{10}$.

${ }^{8}$ Hemos intentado determinar si este ejemplar se encontraba ya incorporado a los fondos más antiguos del Colegio de San Ildefonso. De la revisión de los primeros inventarios conservados se desprenden los siguientes datos:

$\left.1 .^{\circ}\right)$ El más antiguo de ellos, datado en torno a 1512 (Libro becerro de los juros, censos, heneficios, préstamos, rentas, tributos. propiedades, possesiones y señorios del Coleglo, Archivo Histórico Nacional, sign. Universidades-Libro 1090-F, Index omnium librorum bibliothecae collegii Santi Illdefonsi oppidi Complutensi, ff. $33^{r}-98^{v}$ ) se consigna la existencia de unos «Comentarios de Cesar» (f. 92r, lin. 27). El hecho de que se enuncie el título en castellano así como el que aparezca en el contexto de una relación de traducciones induce a pensar que probablemente no se trate de la edición del texto latino, sino quizá de la única traducción al castellano realizada antes de la elaboración de este îndice, que llevó a cabo DiEGo LóPEZ DE DE TOLEDo (Toledo 1498).

$\left.2 .^{\circ}\right)$ El Inventario de los bienes del Colegio mayor de S. Ildefonso de la ciudad de Alcalá (Archivo Histórico Nacional, sign. Universidades-Libro 1091-F), fechado hacia 1523, contiene una Tabula librorum bibliothecae Collegii IIldephonsi (f. $6^{r}-18^{r}$, en la cual se registran unos aComentaria Cesaris» (f. 17', col. b, lin. 21). No es posible determinar si esta única mención remite al manuscrito del Bellum Gallicum conservado actualmente en la Biblioteca Histórica del Marqués del Valdecilla (Universidad Complutense de Madrid), sign. 142, o bien al ejemplar de la edición burgalesa I-275 que se conserva en la misma.

3. ) En el registro de libros de 1526 , incluido en el Inventario de los bienes del Collegio mayor de S. Ildefonso (Archivo Histórico Nacional, sign. Universidades-Libro 1092-F), se incluyen, dentro de la Tabulla librorum bibliothecae Collegii Scti. Illdefonsi (ff. 21'-41') dos referencias: «Comentarios de Gayo Julio Cesar» (f. 40", lin. 1), en el contexto de una relación de traducciones, en alusión, posiblemente a la traducción antes mencionada de Lopez de Toledo de 1491; y unos "Commentaria cesaris» (f. 39r, lin. 24), entre otros textos latinos, que igualmente pueden remitir al manuscrito de la Biblioteca de Valdecilla o a nuestra edición.

$4 .^{\circ}$ ) En el Indice alphabetico de los libros contenidos en esta libreria del Colegio Mayor de $S$. Ildephonso de la universidad de Alcala (Biblioteca Histórica «Marqués de Valdecilla», sign. Ms. 308 ), de principios del s. XVII. se consignan unos «Commentaria Cesaris», pero que no parecen correspoder al ejemplar de la edición de Burgos, sino al manuscrito sign 142 de la misma biblioteca, ya que en este índice se le asigna el registro: "lt/B/25 f.», el mismo que se registra en el explicit de este manuscrito.

De la revisión de estos índices, por tanto, no se puede deducir con seguridad que el ejemplar I275 se encontrara en el fondo antiguo del Colegio de San Ildefonso. Sobre la integración de los manuscritos y libros en esta biblioteca, cf. V. De la Fuente. «Formación y vicisitudes de la Biblioteca Complutense", Boletín-Revista de la Universidad de Madrid II, 1870, pp. 717-721 722-727: 815-823; 1191-1208. Sobre la adquisición de libros en la época de Cisneros, cf. J. Garcia Oro, El Cardenal Cisneros: vida y empresas, II, Madrid 1993. Cf. J. CANTó y A. HuARTE, op. cit. pp. XVIII-XIX. 
Sin embargo, la colación de ambos ejemplares permite comprobar que existe una significativa serie de variantes entre $\mathbf{b}^{\boldsymbol{m}}$ y $\mathbf{b}^{\boldsymbol{c}}$, cuyo análisis es el objeto de nuestro estudio.

\section{LECTURAS DISCREPANTES ENTRE $\mathbf{b}^{\mathbf{m}} \mathbf{y} \mathbf{b}^{\mathbf{c}}$}

La relación de variantes entre uno y otro ejemplar es la siguiente ${ }^{11}$ :

\begin{tabular}{|c|c|c|}
\hline & $\mathbf{b}^{m}$ & $\mathbf{b}^{\mathbf{c}}$ \\
\hline $\mathrm{b}^{\text {lr }(\mathrm{f} .7 \mathrm{r})}$ I. $32($ (B.G. I 40,2) & cupidissime & cupidissime \\
\hline $\mathrm{b}^{2 \mathrm{r}}\left(\mathrm{f} .8^{\mathrm{r}}\right)$ ) $.3(B . G . \mathrm{I} 42,5)$ & eqnitibns & eqnibns \\
\hline $\mathrm{b}^{2 r}($ f. 8 ) I. $5($ B.G. I 42,5) & ligionis & legionis \\
\hline $\mathrm{b}^{2 \mathrm{r}}\left(\mathrm{f} .8^{\mathrm{r}}\right) \mathrm{l} .13($ B.G. I 43,3) & colloquereutur & colloquernetur \\
\hline $\mathrm{b}^{4 \mathrm{r}}\left(\mathrm{f} .10^{r}\right)$ (encabezado) & secundus & secnndus \\
\hline$b^{4 \mathrm{r}}\left(\right.$ f. $\left.10^{r}\right)$ l. $3($ B.G. II 1,3$)$ & $\begin{array}{l}\text { inueterascerein Gallia } \\
\text { moleste ferebant }\end{array}$ & $\begin{array}{l}\text { inueterascere in Gallia } \\
\text { molest(e)ferebant }\end{array}$ \\
\hline
\end{tabular}

${ }^{9}$ Esta edición burgalesa no fue registrada por L. HaIn en su célebre Repertorium Bibliographicum, Stuttgart 1824-1891 (Milán 1948), I, pp. 5-8; cf. W. A. CoPINGER, Supplement to Hain's Repertorium Bibliographicum, Londres 1894, Milán 1950, II, pp. 132-133, n. ${ }^{\circ} 1401$ (delante). La primera referencia en autores hispanos se encuentra en FR. MÉNDEZ, Tipografía española, op. cit., p. 135 (9). Posteriormente C. HAEBLER, Biblioteca ibérica del siglo XV, op. cit., N. ${ }^{\circ} 112$ (p. 50); M. Menéndez Pelayo, Bibliografia Hispano Latina Clásica, op. cit., vol. II. p. 115; Fr. VINDEL. El arte tipográfico en España durante el s. XV (Burgos y Guadalajara), op cit. pp. 60-64 (n. ${ }^{\circ} 20$ ). FR. García Craviotto (coord.), Catálogo General de Incunables en Bibliotecas Españolas, Madrid 1989, p. 245, n. ${ }^{\circ}$ 1585; El Catálogo Colectivo del Patrimonio Bibliográfico Español, del Ministerio de Educación, Cultura y Deporte; Gesamtkatalog der Wiegendrucke VI, Stuttgart-Nueva York 1938 (1968), p. 31, n. ${ }^{\circ} 5875$.

${ }^{10} \mathrm{La}$ coincidencia entre ambos ejemplares puede comprobarse en la descripción general de esta edición incunable: 50 hojas sin numerar, $200 \times 276 \mathrm{~mm}$., impresión en línea tirada con caracteres góticos en dos tamaños, uno para el cuerpo del texto y otro mayor para títulos y colofón (tipos 1:104G, 2:160G). Espacio para iniciales en letras capitales grabadas al comienzo de cada libro. En tinta negra. Consta de ocho cuadernillos: siete de seis hojas cada uno y el último de ocho hojas, marcados con signaturas tipográficas en el ángulo inferior derecho del recto $\left(\mathrm{a}^{6}-\mathrm{g}^{6}, \mathrm{~h}^{8}\right)$. El colofón $(50 \mathrm{v}$ , lín 40-42) reza así: Anno saluatoris nostri $M \cdot C C C C \cdot L X X X X J$ mense apri/li hoc opus summa cum diligentia burgis in officilna ioannis burgiensis impressum est. Cf. A. Moreno Hernández, «La edición incunable del Bellum Gallicum de Julio César de Burgos 1491», art. cit.

1 Tras la identificación de la página del cuadernillo, del folio (entre paréntesis) y de la línea correspondiente a la variante, se indica el pasaje de la obra de César en el cual se encuentra la misma. La edición de referencia es la de W. Hering, C. Iulii Caesaris Commentarii rerum gestarum vol. I. Bellum Gallicum, Teubner, Leipzig 1987. 


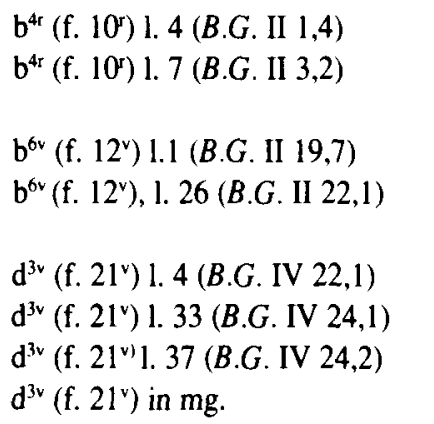

$\mathrm{d}^{4 \mathrm{r}}$ (f. 22r) 1.29 (B.G. IV 27,2)

$\mathrm{d}^{4 \mathrm{r}}$ (f. 22r) 1. 34 (B.G. IV 27,6)

$\mathrm{d}^{4 \mathrm{r}}$ (f. 22r) 1. 37 (B.G. IV 27,7)

$\mathrm{g}^{\text {Iv }}\left(\right.$ f. $\left.37^{\mathrm{v}}\right), 1.9$ (B.G. VI 41,3)

$\mathrm{g}^{2 \mathrm{r}}$ (f. 38 ) 1. 1-6 (Inicial)

$\mathrm{g}^{2 \mathrm{r}}$ (f. 38') l. 1 (B.G. VII 1,1)

$\mathrm{g}^{2 \mathrm{r}}\left(\right.$ f. $\left.38^{5}\right)$ ). $3($ B.G. VII 1,1)

$\mathrm{g}^{2 \mathrm{r}}$ (f. 38') 1. 20 (B.G. VII 2,2)

$\mathrm{g}^{2 \mathrm{r}}$ (f. 38') l. 24 (B.G. VII 3,1)

$\mathrm{g}^{2 \mathrm{r}}$ (f. $38^{r}$ ) l. 39 (B.G. VII 4,3)

$\mathrm{g}^{2 \mathrm{r}}$ (f. $38^{\mathrm{r}}$ ) in $\mathrm{mg}$.

$\mathrm{g}^{3 \mathrm{v}}$ (f. 39v) 1. 4 (B.G. VII 11,8)

$\mathrm{g}^{3 \mathrm{v}}$ (f. 39v) l. 30 (B.G. VII 14,3)

$\mathrm{g}^{4 \mathrm{r}}$ (f. 40') 1. 2 (B.G. VII 14,10)

$\mathrm{g}^{4 \mathrm{r}}\left(\right.$ f. $\left.40^{\circ}\right) 1.5$ (B.G. VII 15,2)

$\mathrm{g}^{4 \mathrm{r}}\left(\right.$ f. $\left.40^{\mathrm{r}}\right)$ l. 8 (B.G. VII 15,4)

$\mathrm{g}^{4 \mathrm{r}}\left(\right.$ f. $\left.40^{\mathrm{r}}\right)$ l. 28 (B.G. VII 17,3)

$\mathrm{g}^{4 \mathrm{r}}$ (f. $\left.40^{r}\right)$ 1. 32 (B.G. VII 17,5)

$\mathrm{g}^{4 \mathrm{r}}$ (f. $\left.40^{r}\right)$ l. 33 (B.G. VII 17,6)

$\mathrm{g}^{4 \mathrm{r}}$ (f. $\left.40^{r}\right)$ l. 35 (B.G. VII 17,8)

$\mathrm{g}^{5 \mathrm{v}}\left(\right.$ f. $\left.41^{\mathrm{v}}\right)$ 1.7-8 (B.G. VII 25,2$)$

$\mathrm{g}^{\text {sv }}$ (f. $\left.41^{\mathrm{v}}\right) 1.10$ (B.G. VII 25,4) a nonnullis etia

permittere

decurreruut

se pibusque densissimi

morinorum

essedariis

one $<r>e$

Caesar Britan/niam at tigit tigit

Britanniam

obsidesque imperauit:

Quorum illi partē statim ciuitates

recepisse

$Q$

Quiecta...cōuentus

coniurare $\bar{t}$

pressetia

Carnutes

quoscunque

Ciues Roma/ni

desideratis

ld esse

seruitutem

magno... proponebāt

pressildio

caruerint

complures

acciperent...lo/co

$\mathrm{Hec}$

ma/nus

ictu ab nonnulis etiā

permitere

decurrerunt

sepibusque désissimis

merinorum

esse dariis

onore

Caesar Britāl tanniam at/

Britauniam

osidesque imperauit:

Quorum illi partem statim citates

recēpisse

om.

uieta...conuentus

coniurarent

pressētia

Caruutes

quoscunque

Ciues Rôalni

desyderatis

Idesse

sernitutem

mano...proponebant

piesildio

carberint

conplures

aciperent..lo-/co

$\mathrm{Hec}$

maluus

inctu 


$\begin{array}{lll}\mathrm{g}^{5 v}\left(\mathrm{f} .41^{v}\right) 1.24(\text { B.G. VII 26,5) } & \text { praeoccuparentur } & \text { prē occuparentur } \\ \mathrm{g}^{5 v}\left(\mathrm{f} .41^{v}\right) 1.29(B . G . \text { VII } 27,2) & \text { cohortatur } & \text { cohortatui } \\ \mathrm{g}^{5 v}\left(\mathrm{f} .41^{v}\right) 1.33-34(\text { B.G. VII 28,1) } & \text { contra ue/niretur } & \text { contraue/niretur } \\ \mathrm{g}^{5 v}\left(\mathrm{f} .41^{v}\right) 1.36(\text { B.G. VII 28,2) } & \text { armis } & \text { armi }\end{array}$

Es sabido que las ediciones de los ss. XV y XVI eran susceptibles de sufrir modificaciones de distinta índole, que los estudios modernos tienden a tipificar en dos grandes grupos: las denominadas 'emisiones' de una edición, que se conciben como una modificación deliberada de una parte de los ejemplares de una misma edición ${ }^{12}$, y los 'estados', esto es, las alteraciones que no responden a un plan intencional y que afectan a parte de los ejemplares de una edición ${ }^{13}$, dos modalidades de variación que se aprecian igualmente en las ediciones de los textos latinos del humanismo español ${ }^{14}$.

Pues bien, en el caso que nos ocupa, el análisis de la índole y disposición de las variantes de los dos ejemplares conservados del incunable burgalés permite extraer las siguientes conclusiones:

12 J. MOLl, «Problemas bibliográficos del libro del siglo de Oro», Boletín de la Real Academia Española 49, 1979, dedica las páginas 59-65 de su trabajo al análisis de las características de las emisiones, que se conciben como «el conjunto de ejemplares, parte de una edición, que forma una unidad intencionalmente planteada»(p. 59).

${ }_{13}$ Así, el estado se refiere «a las variaciones, no planeadas intencionalmente, que presentan los ejemplares de una edición, producidas durante la impresión o posteriormente a la misma o a su puesta en venta», según J. MoLl, ibidem, p. $65-76$ (p.65).

${ }^{14}$ Sobre las frecuentes alteraciones de que eran objeto las ediciones de textos latinos del humanismo español, pueden verse, por ejemplo, los trabajos sobre distintas emisiones de los comentarios de Nebrija a Prudencio (F. F. González VeGA, «El caso de los comentarios de Nebrija a Prudencio y las distintas emisiones de 1512», en J. M. MaEstre y J. Pascual Barea (coords), Humanismo y pervivencia del Mundo Clásico. Actas del 1 Simposio sobre Humanismo y pervivencia del Mundo Clásico, Cádiz 1993, vol. I.1., pp. 495-506); o la detección de al menos tres emisiones diferentes, con modificaciones que afectan primordialmente a la sintaxis y a la semántica. de la primera edición de una obra de Bernardino Gómez Miedes, emisiones identificadas por $\mathbf{S}$. Ramos Maldonado, «Las distintas emisiones de la editio princeps de los Commentariorum de sale libri V de Bernardino Gómez Miedes», ExcPhil IV-V, 1994-1995, pp. 371-392. Otro caso de divergencias de redacción es el que se verifica en una oda de Arias Montano (Amberes 1571, dos ediciones; Amberes 1589; Valencia 1774), debidas básicamente a razones métricas, a las anomalías en la escansión de los endecasílabos alcaicos, y, en menor medida, a la selección léxica, que fueron estudiados por J. L. NAVARro LOPEZ, «Dos versiones diferentes de la oda XI de las $\mathrm{Hu}$ manae salutis monumenta», Excerpta philologica Antonio Holgado Redondo sacra I.2. (1991), pp. 545-563. J.M. Maestre ha analizado las variaciones debidas a autocensura en una obra de Sobrarias, cf. «Limae labor y creación literaria en latín durante el Renacimiento: las dos versiones del Carmen in natali serenissimi Philippi, Hispaniarum principis catholici de Sobrariasm, en J. M. Maestre Maestre y J. Pascual Barea (coords.), Humanismo y pervivencia del mundo clásico, Cádiz 1993, I.1. 135-178. 
a) Las modificaciones se han introducido exclusivamente en 11 páginas, correspondientes a tres pliegos (b, d y g) de los ocho de que consta la edición.

b) Las variantes son fruto de correcciones puntuales introducidas durante el proceso de composición e impresión de cada pliego.

Estas dos circunstancias revelan que las divergencias entre $\mathbf{b}^{\mathbf{m}}$ y $\mathbf{b}^{\mathbf{c}}$ no son fruto de lo que técnicamente se considera como diferentes 'emisiones' de una edición, sino de dos 'estados' de la misma. A pesar de la falta de información documental que tenemos sobre muchos detalles del funcionamiento de las primeras prensas hispanas de tipos móviles, podemos reconstruir con cierta precisión el estadio en el que se introducen estas modificaciones ${ }^{15}$. El original era objeto, por parte de los cajistas o componedores, de la composición tipográfica de los moldes de las páginas de un pliego, según el plegado previsto, y, tras las correcciones pertinentes, se llevaba a cabo la tirada del mismo, que sin embargo podía detenerse, bien por una revisión habitual, bien por advertirse la necesidad de introducir correcciones. Se introducían las modificaciones sobre las planchas y se continuaba con la tirada prevista del pliego, para luego descomponer los moldes, limpiar los tipos e iniciar el proceso para un nuevo pliego. Los ejemplares de la primera tirada normalmente no se destruían, habida cuenta de la escasez y valor del papel, por lo que se incorporaban al libro, dando lugar a la existencia de «estados» distintos de una misma obra. Esta operación puede llegar a afectar a varios pliegos de un mismo libro, variando el número de ejemplares de cada pliego corregidos o sin corregir, según el momento de la tirada en que se advirtió la errata y se paró la prensa para verificar la corrección.

Por consiguiente, dentro de los dos tipos de estados que se distinguen en las ediciones antiguas ${ }^{16}$, las divergencias que se aprecian entre estos dos ejemplares de esta edición incunable burgalesa del Bellum Gallicum de César corresponden a la modalidad de las alteraciones que no afectan a la estructura de la obra, sino que presenta correcciones realizadas durante la tirada que sólo afectan a parte de los ejemplares.

is Sobre este proceso de impresión. cf. J. Moll. ibidem, p. 65-76. A. BlecuA, Manual de critica textual, Madrid 1983, pp. 172-173, G.S. Sosa, Manual de incunables (Historia de la imprenta hasta el s. XVIII), Buenos Aires 1972, pp. 189-218 (sobre la estructura de las prensas del periodo incunable, cf. pp. 207-212), y M. A. PÉREZ PRIEGO, Introducción general a la edición del texto liıerario, Madrid 2001, pp. 61-64. Cf. También los estudios publicados bajo la dirección de Fr. Rico, Imprenta y crítica textual en el siglo de Oro. Valladolid 2000.

is Cf. J. MOLL, op. cit., pp. $65-76$ 


\section{ANÁliSIS DE LAS VARIANTES}

La índole de las divergencias entre $\mathbf{b}^{\mathbf{m}} \mathbf{y} \mathbf{b}^{\mathbf{c}}$ permite distinguir una tipología de variantes que responden a tres niveles distintos:

1. $^{\circ}$ Variantes externas al texto del B.G.

Se trata de alteraciones efectuadas fuera de la caja del texto latino y que afectan a dos elementos:

a) Encabezamientos: en $b^{4 r}\left(\right.$ f. $\left.10^{r}\right) b^{\mathbf{c}}$ presentaba secnndus que $\mathbf{b}^{\mathrm{m}}$ ha corregido en secundus.

b) Títulos marginales: la edición burgalesa, siguiendo las pautas de edición de su modelo, la edición veneciana de $1482^{17}$, ha incorporado las anotaciones marginales de ésta, que en dos ocasiones presentan alteraciones entre los dos ejemplares conservados: en $\mathrm{d}^{3 \mathrm{v}}$ (f. $21^{\mathrm{v}}$ in $\mathrm{mg}$.) $\mathbf{b}^{\mathrm{c}}$ presenta una ditografía (Caesar Britā/tanniam at/tigit), que $\mathbf{b}^{\mathbf{m}}$ corrige (Caesar Britan/niam at tigit), siguiendo a la edición veneciana (v Caesar Bri//tanniam at/tigit); en $\mathrm{g}^{2 \mathrm{r}}$ (f. $38^{\mathrm{r}}$ in $\mathrm{mg}$.) $\mathbf{b}^{\mathbf{c}}$ registra Ciues $R \bar{o} a / n i$, mientras $\mathbf{b}^{m}$ resuelve la abreviatura (Ciues Roma/ni).

2..$^{\circ}$ Impresión de un grabado para la letra inicial de un libro. El grabado de estas letras era un proceso posterior a la tirada del pliego ${ }^{18}$. En la composición se ha previsto un hueco alterando la caja de las primeras líneas para rellenarlo después con el grabado de la letra correspondiente. En el comienzo del libro VII, localizado en $\mathrm{g}^{2 \mathrm{r}}$ (f. 38r,1-6), $\mathbf{b}^{\mathbf{c}}$ conserva el hueco en blanco, mientras $\mathbf{b}^{\mathrm{m}}$ ha incorporado el grabado de la $Q$ inicial. Se trata de una diferencia sustancial en el tratamiento de uno y otro pliego.

$3^{\circ}$ ) Variantes introducidas en el texto. La tipología de lecturas altemativas presenta las siguientes variedades:

17 Se trata, como hemos intentado demostrar en «La edición incunable del Bellum Galli“um de J. César....», art. cit., de la edición impresa en 1482 por Octavianus Scotus Modoetensis en Venecia.

in Sobre la técnica del grabado en los impresos incunables, cf. G. S. SoSA, Manual de incunables, op. cit., pp. 253-271. 
A) Confusión de tipos

a) Confusión $u / n$

\begin{tabular}{|c|c|c|}
\hline $\mathrm{b}^{4 \mathrm{r}}$ (f. $\left.10^{\mathrm{r}}\right)$ (encabezado) & $\mathbf{b}^{\mathbf{m}}$ secundus & $\mathbf{b}^{\mathbf{c}}$ secnndus \\
\hline$b^{6 v}\left(\right.$ f. $\left.12^{v}\right) 1.1$ & $\mathbf{b}^{\mathbf{m}}$ decurreruut & $\mathbf{b}^{\mathbf{c}}$ decurrerunt \\
\hline $\mathrm{d}^{4 r}\left(\right.$ f. $\left.22^{r}\right) 1.29$ & $\mathbf{b}^{\mathbf{m}}$ Britanniam & $\mathbf{b}^{\mathbf{c}}$ Britauniam \\
\hline $\mathrm{g}^{2 \mathrm{r}}\left(\right.$ f. $\left.38^{\mathrm{r}}\right) 1.39$ & $\mathbf{b}^{\mathbf{m}}$ quoscunque & $\mathbf{b}^{c}$ quoscuuque \\
\hline $\mathrm{g}^{4 \mathrm{r}}\left(\right.$ f. $\left.40^{r}\right) 1.2$ & $\mathbf{b}^{\mathrm{m}}$ seruitutem & $\mathbf{b}^{\mathfrak{c}}$ sernitutem \\
\hline $\mathrm{g}^{5 v}\left(\right.$ f. $\left.41^{v}\right) 1.7-8$ & $\mathbf{b}^{\mathrm{m}} \mathrm{ma} /$ nus & $\mathbf{b}^{\mathbf{c}} m a / u u s$ \\
\hline
\end{tabular}

b) Confusión $r / i$
$\mathrm{g}^{4 \mathrm{r}}$ (f. $40^{\mathrm{r}}$ ) 1.8
$\mathbf{b}^{\mathbf{m}}$ presildio
$\mathbf{b}^{\mathbf{c}}$ piessi/dio
$\mathrm{g}^{5 \mathrm{v}}\left(\mathrm{f} .41^{\mathrm{v}}\right) 1.29$
b' $^{\mathbf{m}}$ cohortatur
b $^{\mathbf{c}}$ cohortatui

B) Adición o supresión de uno o varios tipos:
$b^{2 r}\left(f .8^{r}\right), 1.3$
b'm eqnitibns $^{\text {m }}$
bc eqnitibns
$\mathrm{d}^{4 \mathrm{r}}$ (f. $22^{\mathrm{r}}$ ), 1.34
$\mathbf{b}^{\mathbf{m}}$ obsidesque
$b^{c}$ osidesque
imperauit: Quorum
imperauit: Quorum
illi partē statim
$\mathrm{d}^{4 \mathrm{r}}$ (f. $\left.22^{\text {r) }}\right) 1.37$
$\mathbf{b}^{\mathbf{m}}$ ciuitates
illi partem statim
$\mathrm{g}^{2 \mathrm{r}}$ (f. 38 $) 1.1$
$\mathbf{b}^{\mathbf{m}}$ Quiecta...cōuentus
$\mathbf{b}^{\mathbf{c}}$ citates
$\mathrm{g}^{4 \mathrm{r}}\left(\mathrm{f} .40^{r}\right) 1.5$
b $^{\text {m }}$ magno...
$\mathbf{b}^{\mathbf{c}}$ uieta...conuentus
proponebāt

C) Alteración del orden de los tipos
$b^{2 r}\left(\right.$ f. $8^{\text {r) }}$ 1. 13
$\mathbf{b}^{\text {m }}$ colloquereutur
$\mathbf{b}^{\mathbf{c}}$ colloquernetur

D) Signo de nasalización
$b^{4 r}\left(\right.$ f. $\left.10^{r}\right) 1.4$
$\mathbf{b}^{\mathrm{m}}$ etia $\zeta$
$\mathbf{b}^{\mathbf{c}}$ etīa
$\mathrm{g}^{\text {lv }}$ (f. $\left.37^{v}\right) 1.9$
b $^{m}$ recepisse
$b^{c}$ recēpisse
$\mathrm{g}^{2 x}$ (f. 38 $)$ l. 3
$\mathbf{b}^{\mathbf{m}}$ coniurare $\bar{e} t$
b' coniurarent $^{\mathbf{c}}$
$\mathrm{g}^{4 \mathrm{r}}\left(\right.$ f. $\left.40^{\mathrm{r}}\right) \mathrm{l} .5$
$\mathbf{b}^{\mathbf{m}}$ proponebāt
$b^{\mathbf{c}}$ proponebant 
E) Cortes de palabra

\begin{tabular}{|c|c|c|}
\hline$b^{4 r}\left(\right.$ f. $\left.10^{r}\right) 1.3$ & $\begin{array}{l}\mathbf{b}^{\mathbf{m}} \text { inueterascerein } \\
\text { Gallia moleste } \\
\text { ferebant }\end{array}$ & $\begin{array}{l}\mathbf{b}^{\mathbf{c}} \text { inueterascere in } \\
\text { Gallia } \\
\text { molest(e)ferebant }\end{array}$ \\
\hline $\mathrm{d}^{3 \mathrm{v}}\left(\right.$ f. $\left.21^{v}\right) \mathrm{l} .33$ & $\mathbf{b}^{\mathbf{m}}$ essedariis & $\mathbf{b}^{\mathbf{c}}$ esse dariis \\
\hline $\mathrm{g}^{3 \mathrm{v}}\left(\right.$ f. $\left.39^{v}\right) 1.30$ & $\mathbf{b}^{\mathrm{m}}$ Id esse & $\mathbf{b}^{\mathbf{c}}$ Idesse \\
\hline$g^{\text {sv }}\left(\right.$ f. $\left.41^{v}\right)$ l. 24 & $\mathbf{b}^{\mathbf{m}}$ praeoccuparentur & $\mathbf{b}^{\mathbf{c}}$ pre occupar \\
\hline $\mathrm{g}^{5 \mathrm{v}}\left(\mathrm{f} .41^{\mathrm{v}}\right)$ l. $33-34$ & $\mathbf{b}^{\mathrm{m}}$ contra ue/niretur & $\mathbf{b}^{\mathbf{c}}$ contraue/niretur \\
\hline
\end{tabular}

F) Variantes gráfico-fonéticas
$b^{\text {Ir }}\left(\right.$ f. $7^{\text {r }) ~} 1.32$
$\mathbf{b}^{\mathrm{m}}$ cupidissime
$\mathbf{b}^{\mathbf{c}}$ cupidissime
$b^{2 r}\left(\right.$ f. $\left.8^{\text {r }}\right) 1.5$
b' $^{\text {m ligionis }}$
bc legionis
$b^{4 \mathrm{r}}\left(\right.$ f. $\left.10^{\mathrm{r}}\right) 1.17$
$\mathbf{b}^{\mathbf{m}}$ permittere
bc permitere
$\mathrm{g}^{2 \mathrm{r}}\left(\right.$ f. $38^{\text {r) }}$ l. 20
b $^{\text {m }}$ presêtia
b $^{c}$ presētia
$\mathrm{g}^{3 \mathrm{v}}$ (f. $39^{\mathrm{v}}$ ) 1.4
$\mathbf{b}^{\mathbf{m}}$ desideratis
$\mathbf{b}^{\mathbf{c}}$ desyderatis
$\mathrm{g}^{4 \mathrm{r}}\left(\right.$ f. $\left.40^{r}\right) 1.28$
$\mathbf{b}^{\mathbf{m}}$ caruerint
bc carberint
$\mathrm{g}^{4 \mathrm{r}}\left(\right.$ f. $\left.40^{\mathrm{r}}\right)$ l. 32
$b^{\text {m }}$ complures
b' conplures
$\mathrm{g}^{4 \mathrm{r}}\left(\mathrm{f} .40^{\mathrm{r}}\right) 1.33$
$\mathrm{b}^{\mathbf{m}}$ acciperent...lo/co
bc aciperent..lo-/co
$\mathrm{g}^{4 \mathrm{r}}\left(\right.$ f. $\left.40^{\mathrm{r}}\right) 1.35$
b $^{\mathbf{m}} \mathrm{Hec}$
$b^{\mathbf{c}} \mathrm{HeC}$

G) Variantes morfosintácticas
$b^{6 v}\left(\right.$ f. $\left.12^{v}\right) 1.26$
$\mathbf{b}^{\mathrm{m}}$ se pibusque

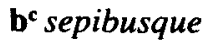
densissimi
$g^{5 v}\left(f .41^{v}\right) 1.36$
$\mathbf{b}^{\mathbf{m}}$ armis
dēsissimis
b $^{\mathbf{c}}$ armi

H) Variantes léxicas
$d^{3 v}$ (f. $\left.21^{v}\right) 1.37$
$\mathbf{b}^{m}$ one $<r>e$
$b^{c}$ onore
$\mathrm{g}^{5 \mathrm{v}}\left(\mathrm{f} .41^{v}\right) 1.10$
$\mathbf{b}^{\mathrm{m}}$ ictu
bc inctu

I) Nombres propios
$\mathrm{d}^{3 \mathrm{v}}$ (f. $\left.21^{\mathrm{v}}\right) 1.4$
$\mathbf{b}^{\mathbf{m}}$ morinorum
b $^{\mathbf{c}}$ merinorum
$\mathrm{g}^{2 \mathrm{r}}\left(\right.$ f. $\left.38^{r}\right) 1.24$
b' $^{\text {m Carnutes }}$
$b^{c}$ Caruutes 


\section{Caracterización de los dos estados}

El examen de estas variantes a la luz de la técnica de elaboración de las ediciones incunables permite extraer algunas conclusiones sobre la índole de los dos estados que presenta esta edición:

1..$^{\circ}$ En tres cuadernillos de los ocho de que consta la edición se han introducido correcciones que corresponden a dos estados distintos de los mismos. Como la encuadernación de los ejemplares es lógicamente posterior a la tirada, hay que determinar a qué estado corresponde cada uno de los pliegos en los dos ejemplares conservados.

a) El pliego b se ve afectado en 4 páginas y un total de 10 variantes, cuyo contraste permite establecer que el estado más antiguo es el que se conserva en $\mathbf{b}^{\mathbf{c}}$, sobre el cual se han introducido correcciones de imprenta y variantes grafico-fonéticas y morfológicas que han quedado registradas en el pliego que conserva $\mathbf{b}^{\mathrm{m}}$.

b) El pliego d ha sido corregido sólamente en 2 páginas, que presentan 7 variantes, de cuyo examen se desprende que el cuadernillo tirado inicialmente es el de $\mathbf{b}^{\mathbf{c}}$, ya que, en todos los casos, se trata de lecturas revisadas en la segunda tirada del pliego, incorporado $\mathbf{a ~}^{\mathbf{m}}$, donde se corrigen erratas de imprenta y se introducen correcciones gráfico-fonéticas, morfosintácticas, léxicas y nombres propios.

c) El tercer pliego con correcciones (g), presenta 5 páginas con variantes, que suman en total 23 lecturas discrepantes, en las cuales puede advertirse nítidamente que la tirada inicial es la conservada en el pliego del ejemplar $\mathbf{b}^{\mathbf{c}}$, que ha sido objeto, en la segunda tirada, preservada en el cuadernillo de $\mathbf{b}^{\mathbf{m}}$, de una amplia gama de correcciones, lapsus preli, y variantes del texto latino a todos los niveles (gráfico-fonético, morfosintáctico, léxico y de nombres propios). La ausencia en $\mathbf{b}^{\mathbf{c}}$ del grabado de la letra inicial del comienzo del libro VII en $g^{2 r}$ (f. 38) 1. 1-6, corrobora la secuencia entre el primer y el segundo estado, representado por $\mathbf{b}^{\mathbf{m}}$.

Estos datos pueden sintetizarse en el siguiente cuadro:

\begin{tabular}{clccc}
\hline Pliegos & \multicolumn{1}{c}{$\begin{array}{c}\text { Páginas con } \\
\text { variantes }\end{array}$} & Variantes & $\begin{array}{c}\text { Primer } \\
\text { estado }\end{array}$ & $\begin{array}{c}\text { Segundo } \\
\text { estado }\end{array}$ \\
\hline $\mathrm{b}$ & $4\left(\mathrm{~b}^{1 \mathrm{r}} ; \mathrm{b}^{2 \mathrm{r}} ; \mathrm{b}^{4 \mathrm{r}} ; \mathrm{b}^{6 \mathrm{v})}\right.$ & 10 & $\mathbf{b}^{\mathbf{c}}$ & $\mathbf{b}^{\mathbf{m}}$ \\
\hline $\mathrm{d}$ & $2\left(\mathrm{~d}^{3 \mathrm{v}} ; \mathrm{d}^{4 \mathrm{r}}\right)$ & 7 & $\mathbf{b}^{\mathbf{c}}$ & $\mathbf{b}^{\mathbf{m}}$ \\
\hline $\mathrm{g}$ & $5\left(\mathrm{~g}^{1 \mathrm{v}} ; \mathrm{g}^{2 \mathrm{r}} ; \mathrm{g}^{3 \mathrm{v}} ; \mathrm{g}^{4 \mathrm{r}} ; \mathrm{g}^{5 \mathrm{v}}\right)$ & 23 & $\mathbf{b}^{\mathbf{c}}$ & $\mathbf{b}^{\mathbf{m}}$ \\
\hline
\end{tabular}


A pesar de la aparente identidad externa de los dos ejemplares, la presencia en $\mathbf{b}^{\boldsymbol{c}}$ de los tres pliegos correspondientes a la primera tirada del texto latino del Bellum Gallicum convierte a este ejemplar en el exponente del estado inicial de la impresión de la obra, mientras que $\mathbf{b}^{\mathbf{m}}$ registra la versión corregida - y probablemente definitiva-, de la misma, es decir, el segundo estado de la edición que salió de la prensa de Juan de Burgos. La existencia de estos dos ejemplares responde, probablemente, a la circulación de ejemplares en uno y otro estado, ya que, como era habitual en la época, los pliegos iniciales no se eliminaban, ante la carestía de papel. Igualmente resulta significativa la coincidencia de los tres pliegos de cada estado encuadernado en el mismo ejemplar, lo que hace pensar en que la encuadernación de los cuadernillos no se hizo de manera aleatoria -como también se aprecia en ocasiones en los impresos de los ss. XV y XVI-, sino que el impresor ha intentado, deliberadamente, distinguir entre ejemplares con estados distintos del texto.

$2^{\circ}$ ) Las variantes son alteraciones de la composición tipográfica que se restringen a la línea del texto, evitándose introducir modificaciones que impliquen el reajuste de más de una línea. Como consecuencia de ello, las correcciones que exigen la adición o supresión de uno o varios tipos llevan consigo el reajuste dentro de la misma línea. El procedimiento más habitual en estos casos es la compensación de la pérdida o ganancia de espacio a través del tratamiento abreviado o no de las nasales:

$$
\begin{aligned}
& \mathrm{d}^{4 r} \text { (f. } 22^{r} \text { ) } 1.24 \quad \text { b }^{m} \text { obsidesque imperauit: Quorum illi parte } \bar{e} \text { statim } \\
& \text { b }^{\mathbf{c}} \text { osidesque imperauit: Quorum illi partem statim } \\
& \mathrm{g}^{4 \mathrm{r}}\left(\mathrm{f} .40^{\mathrm{r}}\right) \mathrm{l} .5 \quad \mathbf{b}^{\mathrm{m}} \text { magno... proponebāt } \\
& \text { b }^{\mathbf{c}} \text { mano...proponebant }
\end{aligned}
$$

En estos dos casos, el proceso ha sido el mismo: $\mathbf{b}^{\mathbf{m}}$ ha corregido una variante de $\mathbf{b}^{\mathbf{c}}$ introduciendo un tipo nuevo en la línea (obsides por osides; magno por mano) y compensado seguidamente esta adición con la sustitución de una nasal por la vírgula correspondiente (parte por partem; proponebāt por proponebant)

El proceso inverso puede apreciarse en estos ejemplos:

$$
\begin{aligned}
& b^{4 r}\left(\text { f. } 10^{r}\right) 1.4 \\
& \mathbf{b}^{\mathbf{m}} \text { a nonnullis etia } \zeta \\
& \left.b^{\text {tv }} \text { (f. } 12^{v}\right) 1.26 \\
& \mathbf{b}^{\mathbf{m}} \text { densissimi } \\
& \mathbf{b}^{\mathbf{c}} \text { ab nonnulis etiā } \\
& \mathbf{b}^{\mathbf{c}} \text { dêsissimis }
\end{aligned}
$$


La eliminación de un espacio al suprimir un tipo ( $a$ por $a b$; densissimi por désissimis) se compensa mediante el registro de una nasal en lugar de la abre-

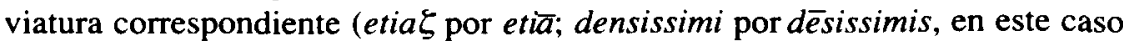
dentro de la misma palabra).

3. ${ }^{\circ}$ Conviene también reparar en los criterios que subyacen en las modificaciones introducidas por el corrector del taller burgalés entre uno y otro estado de la edición.

$\mathrm{Al}$ respecto hay un primer hecho revelador: el volumen de correcciones es extraordinariamente reducido respecto al gran número de lecturas separativas introducidas en la edición burgalesa, no sólo por afectar tan sólo a tres de los ocho cuadernillos, y a 11 de las 100 páginas de la edición, sino porque estas mismas páginas presentan otras muchas variantes que no son objeto de revisión, y la intervención del corrector se limita a introducir modificaciones que no rebasan nunca el ajuste de línea, como ya hemos indicado. En este sentido parece que la labor del corrector de la primera tirada es parcial y hasta cierto punto descuidada, habida cuenta del escaso calado de su trabajo, motivado, posiblemente, por la premura con la que debía revisar los pliegos salidos de la prensa, ya que la introducción de las correcciones implicaba una nueva intervención del cajista y componedor y una probable parada de la prensa hasta completar la tirada del pliego. Esto explicaría también la imprecisión de algunas correcciones, que dan lugar a nuevas erratas, como evidencian estos ejemplos:

- En $b^{2 r}(f .8$ ) l. 13 la lectura del primer estado colloquernetur (b) es enmendada en colloquereutur $\left(\mathbf{b}^{\mathrm{m}}\right)$, que introduce una inversión habitual de los tipos $n / u$.

- El deseo de introducir el tipo que falta en $b^{2 r}\left(\right.$ f. $\left.8^{r}\right)$ l. 3, donde $b^{c}$ presenta eqnitibns, no afecta a los otros dos errores tipográficos que presenta esa misma palabra, y $\mathbf{b}^{\mathbf{m}}$ registra eqnitibns.

- En $\mathrm{d}^{3 \mathrm{v}}\left(\right.$ f. $21^{\mathrm{v}}$ ), 1. 37 la variante onore de $\mathbf{b}^{\mathrm{c}}$ es revisada en el segundo estado de la edición por one $<r>e$, de acuerdo con la lectura de la edición de Venecia 1482, si bien el molde del tipo de la $r$ se ha perdido (one e), dejando el consiguiente hueco en la impresión del pliego.

Pues bien, el criterio básico de las lecturas introducidas en $\mathbf{b}^{\mathbf{m}}$ es la voluntad de corregir las erratas tipográficas que se habían deslizado en $\mathbf{b}^{\mathbf{c}}$ así como de revisar variantes latinas a todos los niveles gramaticales (grafía y fonética, morfología, sintaxis, léxico, cortes de palabra). Estas alternativas podrían, aparentemente, responder a la iniciativa del corrector, si bien en la mayoría de los 
casos están motivadas por el deseo de reproducir fielmente la edición que sirve de base al impreso burgalés, es decir, la edición veneciana de 1482 .

En efecto, de las 40 variantes consignadas en $\mathbf{b}^{m}$, la mayoría de ellas (36) encuentra refrendo en esta edición italiana, hasta el punto de que incluso algunas de las variantes de $\mathbf{b}^{\mathbf{c}}$ se explican como fruto de una lectura errónea del incunable veneciano que luego ha sido enmendada en $\mathbf{b}^{\mathrm{m}}$ : es el caso de $\mathrm{g}^{\mathrm{sv}}$ (f. $41^{\mathrm{v}}$ ) 1.10 , donde la lectura inctu de $b^{c}$ se debe a que en la edición italiana se registra un $i c t u$ con un punto muy denso sobre la $i$ que el tipógrafo del taller burgalés ha confundido con una marca de nasalización, que luego se ha corregido acertadamente en la revisión del pliego.

Las cuatro variantes en las que se verifica el fenómeno inverso, es decir, que $\mathbf{b}^{c}$ sigue a la fuente italiana frente $\mathbf{a} \mathbf{b}^{\mathbf{m}}$, son éstas:

$\begin{array}{lll}\mathbf{b}^{2 \mathrm{r}}\left(\mathrm{f} .8^{\mathrm{r}}\right), 1.5 & \mathbf{b}^{\mathrm{m}} \text { ligionis } & \mathbf{b}^{\mathbf{c}} \text { legionis } \\ \mathrm{b}^{4 \mathrm{r}}\left(\mathrm{f} .10^{\mathrm{r}}\right) 1.4 & \mathbf{b}^{\mathbf{m}} \text { a nonnullis etia\} } & \mathbf{b}^{\mathbf{c}} \text { ab nonnulis etiā } \\ \mathrm{g}^{2 \mathrm{r}}\left(\mathrm{f} .38^{\mathrm{r}}\right) 1.1 & \mathbf{b}^{\mathrm{m}} \text { Quiecta } & \mathbf{b}^{\mathbf{c}}[\text { ]uieta } \\ \mathrm{g}^{3 \mathrm{v}}\left(\mathrm{f} .39^{\mathrm{v}}\right), 1.4 & \mathbf{b}^{\mathbf{m}} \text { desideratis } & \mathbf{b}^{\mathbf{c}} \text { desyderatis }\end{array}$

Estas lecturas revelan que el criterio básico de acomodación a las lecturas del modelo veneciano, a pesar de aplicarse de forma claramente dominante, no impide que el corrector introduzca ocasionalmente variantes que se apartan de su modelo, dejando ver sus preferencias sobre cuestiones gráficas y fonéticas (ligionis, desideratis) o, como en $\mathrm{b}^{4 \mathrm{r}}\left(\mathrm{f} .10^{\mathrm{r}}\right) 1.4$, inclinándose por a nonnullis por analogía con la misma variante que se documenta en la 1.1 de la misma página.

El sentido, pues, de estas correcciones, responde en última instancia a la voluntad del impresor de ceñirse a la edición veneciana de 1482 , dando lugar a la existencia de dos estados de la misma obra impresa, cuyas divergencias responden a una corrección parcial y no a una revisión sistemática de la primera impresión del texto, pero que presenta una amplia gama de variantes cuya inserción se explica, como hemos intentado hacer ver, a partir de los procedimientos y técnicas de las primeras ediciones tipográficas de textos clásicos latinos en el periodo incunable español. 